\title{
Spatial and temporal country-wide survey of temephos resistance in Brazilian populations of Aedes aegypti
}

\author{
Mateus Chediak', Fabiano G Pimenta Jr², Giovanini E Coelho ${ }^{3}$, Ima A Braga ${ }^{3,4}$, \\ José Bento P Lima $^{5}$, Karina Ribeiro LJ Cavalcante ${ }^{6}$, Lindemberg C de Sousa ${ }^{7}$, \\ Maria Alice V de Melo-Santos ${ }^{8}$, Maria de Lourdes da G Macoris ${ }^{9}$, Ana Paula de Araújo ${ }^{8}$, \\ Constância Flávia J Ayres ${ }^{8}$, Maria Teresa M Andrighetti ${ }^{9}$, \\ Ricristhi Gonçalves de A Gomes ${ }^{7}$, Kauara B Campos ${ }^{3}$, Raul Narciso C Guedes ${ }^{1 /+}$
}

\author{
${ }^{1}$ Universidade Federal de Viçosa, Departamento de Entomologia, Viçosa, MG, Brasil \\ ${ }^{2}$ Secretaria Municipal de Saúde de Belo Horizonte, Belo Horizonte, MG, Brasil \\ ${ }^{3}$ Ministério da Saúde, Secretaria de Vigilância em Saúde, Coordenação Geral do Programa Nacional de Controle da Dengue, Brasília, DF, Brasil \\ ${ }^{4}$ Secretaria Municipal de Saúde de São Domingos do Prata, São Domingos do Prata, MG, Brasil \\ ${ }^{5}$ Fundação Oswaldo Cruz, Instituto Oswaldo Cruz, Laboratório de Fisiologia e Controle de Artrópodes Vetores, Rio de Janeiro, RJ, Brasil \\ ${ }^{6}$ Ministério da Saúde, Secretaria de Vigilância em Saúde, Coordenação Geral de Laboratórios de Saúde Pública, Brasília, DF, Brasil \\ ${ }^{7}$ Secretaria de Saúde do Ceará, Núcleo de Controle de Vetores, Laboratório de Entomologia, Fortaleza, CE, Brasil \\ ${ }^{8}$ Fundação Oswaldo Cruz, Centro de Pesquisas Aggeu Magalhães, Recife, PE, Brasil \\ ${ }^{9}$ Secretaria de Saúde de São Paulo, Superintendência de Controle de Endemias, Marília, SP, Brasil
}

\begin{abstract}
The organophosphate temephos has been the main insecticide used against larvae of the dengue and yellow fever mosquito (Aedes aegypti) in Brazil since the mid-1980s. Reports of resistance date back to 1995; however, no systematic reports of widespread temephos resistance have occurred to date. As resistance investigation is paramount for strategic decision-making by health officials, our objective here was to investigate the spatial and temporal spread of temephos resistance in Ae. aegypti in Brazil for the last 12 years using discriminating temephos concentrations and the bioassay protocols of the World Health Organization. The mortality results obtained were subjected to spatial analysis for distance interpolation using semi-variance models to generate maps that depict the spread of temephos resistance in Brazil since 1999. The problem has been expanding. Since 2002-2003, approximately half the country has exhibited mosquito populations resistant to temephos. The frequency of temephos resistance and, likely, control failures, which start when the insecticide mortality level drops below 80\%, has increased even further since 2004. Few parts of Brazil are able to achieve the target $80 \%$ efficacy threshold by 2010/2011, resulting in a significant risk of control failure by temephos in most of the country. The widespread resistance to temephos in Brazilian Ae. aegypti populations greatly compromise effective mosquito control efforts using this insecticide and indicates the urgent need to identify alternative insecticides aided by the preventive elimination of potential mosquito breeding sites.
\end{abstract}

Key words: insecticide resistance survey - dengue - distance interpolation - distribution maps - mosquito larvae

Vector-borne neglected (tropical) diseases such as dengue are an increasing worldwide issue of concern, particularly given current rates of urbanisation, international travel and trade, and climate change, all of which favor the spread of such diseases and their vectors (Hsieh \& Chen 2009, Guzman et al. 2010, Gubler 2012). Mass gatherings and large sporting events are also associated with higher risks of health incidents. The 2014 FIFA World Cup held in Brazil is an example that drew attention and incited debate that focused particularly on dengue due to potential vector outbreaks (Hay 2013). The concern is understandable and justifiable, even if the

doi: 10.1590/0074-02760150409

Financial support: Brazilian Ministry of Health (National Program of Dengue Control from the Office of Health Surveillance).

+ Corresponding author: guedes@ufv.br

Received 23 October 2015

Accepted 17 March 2016 risks were generally small (Lowe et al. 2014, van Panhuis et al. 2014). As a result, no serious incident came to past. The 2016 Olympic Games to be held in Rio de Janeiro are bound to draw a similar level of international attention.

The lack of effective vaccines or pharmaceutical treatments for dengue, typical of the neglected diseases, places mosquito vector control in the forefront of prevention efforts for this disease (Gubler 2004, Halstead 2012). This scenario prevails throughout the affected tropical and subtropical regions of the world, which roughly encompasses about half of the global population (Guzman et al. 2010). Control of the dengue mosquito vector [Aedes aegypti (L.)], which also transmits chikungunya, zika and yellow fever (YF) (thus the common name "yellow fever mosquito"), relies heavily on insecticide use - but there are few compounds available and their use is usually guided by the countries' health officials (OPAS 1995, Funasa 2001, 2002, Braga \& Valle 2007, Araújo et al. 2013, Macoris et al. 2014, Tomé et al. 2014).

The organophosphate temephos is globally the most commonly used insecticide against mosquito larvae due to its high efficacy, low cost and low vertebrate toxic- 
ity (WHO 2009). The result of this overreliance on temephos in controlling YF mosquito larvae is evolution and spread of temephos resistance among populations of this pest species. Such resistance has been detected in various countries since 1995 (Macoris et al. 1995, Mazzari \& Georghiou 1995, Rawlins \& Wan 1995, Bisset Lazcano et al. 2009, Melo-Santos et al. 2010, Bisset et al. 2013, Grisales et al. 2013). Furthermore, the use of temephos for the control of larvae of Ae. aegypti also apparently led to incidental selection for temephos resistance in co-occurring mosquito species populations (Campos \& Andrade 2003, Alves et al. 2011, Phophiro et al. 2011, Amorim et al. 2013), as has also been reported among other co-occurring arthropod pest species (Guedes et al. 2016).

Routine applications of temephos against mosquito larvae in Brazil began in the 1980s (Funasa 1994, 2001, Sucen 1997). The initial suppression of Ae. aegypti in Brazil by 1955 was followed by its subsequent return in the 1970s (Schatzmayr 2000, Lourenço-de-Oliveira et al. 2004). Dengue became endemic in the country and has become an increasingly serious problem since 1986 despite established vector control programs in the country that still continue today (Lourenço-de-Oliveira et al. 2004, Maciel-de-Freitas et al. 2014). By the 1990s, concern emerged in Brazil regarding likely control failures and detection of temephos-resistant mosquito populations, which led to systematic surveys of insecticide resistance in the country and a series of reports on the phenomenon (Macoris et al. 1995, 2003, 2007, 2014, Campos \& Andrade 2003, Lima et al. 2003, 2006, Melo-Santos et al. 2010, Gambarra et al. 2013, Diniz et al. 2014).

A few studies on the underlying mechanisms of temephos resistance followed the initial detection of this phenomenon in Brazil. Despite of an initial report of altered (acetylcholinesterase) target site sensitivity detected in a Brazilian population of Ae. aegypti resistant to temephos from Uberlândia (MG), current evidence suggests the prevalence of enhanced detoxification by metabolising enzymes in an apparently mixed pattern (Braga \& Valle 2007, Melo-Santos et al. 2010, Lima et al. 2011, Gambarra et al. 2013). Congruent findings have been reported from other countries as well (Bisset Lazcano et al. 2009, Bisset et al. 2013, Grisales et al. 2013). Furthermore, recent transcriptome (i.e., the set of all mRNA molecules from a cell) evidence indicates upregulation of detoxification enzymes in insecticide-resistant mosquitoes (Reyes-Solis et al. 2014, Saavedra-Rodriguez et al. 2014). These findings reinforce the perception that multiple metabolic genes are involved in temephos resistance in Ae. aegypti, but with the prevalence of esterase rather than glutathione- $S$-transferase gene expression (ReyesSolis et al. 2014, Saavedra-Rodriguez et al. 2014).

Temephos resistance monitoring in populations of the YF mosquito were underway in Brazil by the late 1990s in response to the increasing incidence of dengue in the country (Braga \& Valle 2007). Scientific reports of the incidence of temephos resistance have increased since then (Campos \& Andrade 2003, Lima et al. 2003, 2006, Macoris et al. 2003, 2007, Melo-Santos et al. 2010, Gambarra et al. 2013, Diniz et al. 2014), but no comprehensive dataset is currently available and no area-wide description of the phenomenon of temephos resistance and its spread has been attempted despite the strategic importance of such information in guiding control policies, protocols and decision-making by Brazilian health officials. The current effort took advantage of the dataset gathered by the National Network of Insecticide Resistance Monitoring (MoReNAa) in Ae. aegypti under the tutelage of the National Program of Dengue Control from the Office of Health Surveillance of the Brazilian Ministry of Health (Brasília, DF, Brazil). The objective of our study was to recognise the spatial and temporal spread of temephos resistance in Brazil for the past 12 years, which we hypothesized, has been acute and has likely encompassed the entire country since 2010.

Our spatial and temporal survey of temephos resistance was performed using standardised procedures for insect sampling and temephos bioassays from the WHO (1981) that were countersigned by the laboratories involved (from MoReNAa) with the support of the Centers for Disease Control and Prevention (CCD, USA), PanAmerican Health Organization and the World Health Organization (Braga et al. 2004, Macoris et al. 2005, Braga \& Valle 2007). The data obtained was subjected to kriging to select suitable semivariogram models for distance interpolation with the goal of generating geospatial maps of the frequency of temephos resistance in Brazilian populations of Ae. aegypti.

\section{MATERIALS AND METHODS}

Insects and insecticide - Mosquito populations were sampled through the MoReNAa (Table I, Fig. 1) as described by Macoris et al. (2003). Briefly, between 100-200 oviposition traps (i.e., ovitraps) were used for this purpose in each city. The ovitraps were placed outdoors in a grid pattern for four weeks, always in the second semester of each year (Fay \& Eliason 1966, Jakob \& Bevier 1969, Funasa 1999). Egg clutches thus collected were used to establish laboratory colonies of over 3,000 individuals from each city (i.e., sampling site). First-generation larvae raised in the laboratory were used in the bioassays (Lima et al. 2003, Macoris et al. 2003). Technical grade temephos (> $90 \%$ pure) was obtained from the Brazilian Ministry of Health and diluted with acetone at the desired concentration for subsequent use in the diagnostic bioassays.

Diagnostic bioassays of temephos resistance - The diagnostic bioassays were performed following the standardised procedures of the WHO $(1981,1992)$. The concentration of temephos required to identify resistant insects (i.e., the diagnostic concentration) was initially established as $14.0 \mu \mathrm{g}$ a.i./L but was subjected to yearly calibration and validation with the standard susceptible Rockefeller strain, as described by Braga et al. (2004) and Macoris et al. (2005). The diagnostic concentration was applied as a $1 \mathrm{~mL}$ solution to each of the experimental containers, reaching a final $250 \mathrm{~mL}$ volume of contaminated water solution (except for the controls, for which only $1 \mathrm{~mL}$ acetone was used). Deionised and distilled water were used to prepare the bioassay solutions. Twenty-five individuals (3rd-4th instar mosquito larvae) were placed in $250 \mathrm{~mL}$ transparent glass contain- 
TABLE I

Sample site identification and geographical coordinates of collection sites for populations of the yellow fever mosquito Aedes aegypti used in the spatio-temporal survey of temephos resistance in Brazil

\begin{tabular}{|c|c|c|c|c|}
\hline Region & State & City & Longitude & Latitude \\
\hline North & Rondônia (RO) & Cacoal & $-61,447222$ & $-11,438611$ \\
\hline North & Rondônia (RO) & Guajará-Mirim & $-65,339444$ & $-10,782778$ \\
\hline North & Rondônia (RO) & Porto Velho & $-63,903889$ & $-8,761944$ \\
\hline North & Rondônia (RO) & Jaru & $-62,466389$ & $-10,438889$ \\
\hline North & Rondônia (RO) & Vilhena & $-60,145833$ & $-12,740556$ \\
\hline North & Acre (AC) & Rio Branco & $-67,810000$ & $-9,974722$ \\
\hline North & Amazonas (AM) & Manaus & $-60,025000$ & $-3,101944$ \\
\hline North & Roraima (RR) & Boa Vista & $-60,673333$ & 2,819722 \\
\hline North & Pará (PA) & Ananindeua & $-48,372222$ & $-1,365556$ \\
\hline North & Pará (PA) & Belém & $-48,504444$ & $-1,455833$ \\
\hline North & Pará (PA) & Benevides & $-48,244722$ & $-1,361389$ \\
\hline North & Pará (PA) & Dom Elizeu & $-47,505000$ & $-4,285000$ \\
\hline North & Pará (PA) & Marabá & $-49,117778$ & $-5,368611$ \\
\hline North & Pará (PA) & Marituba & $-48,341944$ & $-1,355278$ \\
\hline North & Pará (PA) & Rondon do Pará & $-48,067222$ & $-4,776111$ \\
\hline North & Pará (PA) & Sta. Bárbara do Pará & $-48,294444$ & $-1,223611$ \\
\hline North & Pará (PA) & Santarém & $-54,708333$ & $-2,443056$ \\
\hline North & Pará (PA) & Tucuruí & $-49,672500$ & $-3,766111$ \\
\hline North & Amapá (AP) & Macapá & $-51,066389$ & 0,038889 \\
\hline North & Tocantins (TO) & Araguaína & $-48,207222$ & $-7,191111$ \\
\hline North & Tocantins (TO) & Palmas & $-48,360278$ & $-10,212778$ \\
\hline Northeast & Maranhão (MA) & Bacabal & $-44,791667$ & $-4,291667$ \\
\hline Northeast & Maranhão (MA) & São Luís & $-44,302778$ & $-2,529722$ \\
\hline Northeast & Piauí (PI) & Parnaíba & $-41,776667$ & $-2,904722$ \\
\hline Northeast & Piauí (PI) & Teresina & $-42,801944$ & $-5,089167$ \\
\hline Northeast & Ceará (CE) & Caucaia & $-38,653056$ & $-3,736111$ \\
\hline Northeast & Ceará (CE) & Fortaleza & $-38,543056$ & $-3,717222$ \\
\hline Northeast & Ceará (CE) & Juazeiro do Norte & $-39,315278$ & $-7,213056$ \\
\hline Northeast & Rio Grande do Norte (RN) & Caicó & $-37,097778$ & $-6,458333$ \\
\hline Northeast & Rio Grande do Norte (RN) & Jardim do Seridó & $-36,774444$ & $-6,584444$ \\
\hline Northeast & Rio Grande do Norte (RN) & Parnamirim & $-35,262778$ & $-5,915556$ \\
\hline Northeast & Rio Grande do Norte (RN) & Mossoró & $-37,344167$ & $-5,187500$ \\
\hline Northeast & Rio Grande do Norte (RN) & Natal & $-35,209444$ & $-5,795000$ \\
\hline Northeast & Rio Grande do Norte (RN) & Pau dos Ferros & $-38,204444$ & $-6,109167$ \\
\hline Northeast & Paraíba (PB) & Alagoa Grande & $-35,630000$ & $-7,158333$ \\
\hline Northeast & Paraíba (PB) & Bayeux & $-34,932222$ & $-7,125000$ \\
\hline Northeast & Paraíba (PB) & João Pessoa & $-34,863056$ & $-7,115000$ \\
\hline Northeast & Paraíba (PB) & Santa Rita & $-34,978056$ & $-7,113889$ \\
\hline Northeast & Paraíba (PB) & Souza & $-38,228056$ & $-6,759167$ \\
\hline Northeast & Pernambuco (PE) & Araripina & $-40,498333$ & $-7,576111$ \\
\hline Northeast & Pernambuco (PE) & Cabo de Sto Agostinho & $-35,035000$ & $-8,286667$ \\
\hline Northeast & Pernambuco (PE) & Jaboatão dos Guararapes & $-35,014722$ & $-8,112778$ \\
\hline Northeast & Pernambuco (PE) & Moreno & $-35,092222$ & $-8,118611$ \\
\hline Northeast & Pernambuco (PE) & Olinda & $-34,855278$ & $-8,008889$ \\
\hline Northeast & Pernambuco (PE) & Petrolina & $-40,500833$ & $-9,398611$ \\
\hline Northeast & Pernambuco (PE) & Recife & $-34,881111$ & $-8,053889$ \\
\hline Northeast & Pernambuco (PE) & Tamandaré & $-35,104722$ & $-8,759722$ \\
\hline Northeast & Alagoas (AL) & Arapiraca & $-36,661111$ & $-9,752500$ \\
\hline Northeast & Alagoas (AL) & Maceió & $-35,735278$ & $-9,665833$ \\
\hline Northeast & Sergipe (SE) & Aracaju & $-37,071667$ & $-10,911111$ \\
\hline
\end{tabular}




\begin{tabular}{|c|c|c|c|c|}
\hline Region & State & City & Longitude & Latitude \\
\hline Northeast & Sergipe (SE) & Barra dos Coqueiros & $-37,038611$ & $-10,908889$ \\
\hline Northeast & Sergipe (SE) & Itabaiana & $-37,425278$ & $-10,685000$ \\
\hline Northeast & Bahia (BA) & Barreiras & $-44,990000$ & $-12,152778$ \\
\hline Northeast & Bahia (BA) & Eunápolis & $-39,580278$ & $-16,377500$ \\
\hline Northeast & Bahia (BA) & Feira de Santana & $-38,966667$ & $-12,266667$ \\
\hline Northeast & Bahia (BA) & Ilhéus & $-39,049444$ & $-14,788889$ \\
\hline Northeast & Bahia (BA) & Itabuna & $-39,280278$ & $-14,785556$ \\
\hline Northeast & Bahia (BA) & Jacobina & $-40,518333$ & $-11,180556$ \\
\hline Northeast & Bahia (BA) & Jequié & $-40,083611$ & $-13,857500$ \\
\hline Northeast & Bahia (BA) & Potiguará & $-39,876667$ & $-15,594722$ \\
\hline Northeast & Bahia (BA) & Salvador & $-38,510833$ & $-12,971111$ \\
\hline Northeast & Bahia (BA) & Teixeira de Freitas & $-39,741944$ & $-17,535000$ \\
\hline Northeast & Bahia (BA) & Vitória da Conquista & $-40,839444$ & $-14,866111$ \\
\hline Midwest & Mato Grosso do Sul (MS) & Campo Grande & $-54,646389$ & $-20,442778$ \\
\hline Midwest & Mato Grosso do Sul (MS) & Corumbá & $-57,653333$ & $-19,009167$ \\
\hline Midwest & Mato Grosso do Sul (MS) & Coxim & $-54,760000$ & $-18,506667$ \\
\hline Midwest & Mato Grosso do Sul (MS) & Três Lagoas & $-51,678333$ & $-20,751111$ \\
\hline Midwest & Mato Grosso do Sul (MS) & Ponta Porã & $-55,725556$ & $-22,536111$ \\
\hline Midwest & Mato Grosso do Sul (MS) & Dourados & $-54,805556$ & $-22,221111$ \\
\hline Midwest & Mato Grosso (MT) & Cuiabá & $-56,096667$ & $-15,596111$ \\
\hline Midwest & Mato Grosso (MT) & Várzea Grande & $-56,132500$ & $-15,646667$ \\
\hline Midwest & Goiás (GO) & Aparecida de Goiânia & $-49,243889$ & $-16,823333$ \\
\hline Midwest & Goiás (GO) & Goiânia & $-49,253889$ & $-16,678611$ \\
\hline Midwest & Goiás (GO) & Itumbiara & $-49,215278$ & $-18,419167$ \\
\hline Midwest & Goiás (GO) & Luziânia & $-47,950278$ & $-16,252500$ \\
\hline Midwest & Goiás (GO) & Novo Gama & $-48,039444$ & $-16,059167$ \\
\hline Midwest & Goiás (GO) & Rio Verde & $-50,928056$ & $-17,798056$ \\
\hline Midwest & Goiás (GO) & Uruaçu & $-49,140833$ & $-14,524722$ \\
\hline Midwest & Distrito Federal (DF) & Brasília & $-47,929722$ & $-15,779722$ \\
\hline Southeast & Minas Gerais (MG) & Belo Horizonte & $-43,937778$ & $-19,920833$ \\
\hline Southeast & Minas Gerais (MG) & Formiga & $-45,426389$ & $-20,464444$ \\
\hline Southeast & Minas Gerais (MG) & Januária & $-44,361667$ & $-15,488056$ \\
\hline Southeast & Minas Gerais (MG) & Montes Claros & $-43,861667$ & $-16,735000$ \\
\hline Southeast & Minas Gerais (MG) & Teófilo Otoni & $-41,505278$ & $-17,857500$ \\
\hline Southeast & Minas Gerais (MG) & Ubá & $-42,942778$ & $-21,120000$ \\
\hline Southeast & Minas Gerais (MG) & Uberaba & $-47,931944$ & $-19,748333$ \\
\hline Southeast & Minas Gerais (MG) & Uberlândia & $-48,277222$ & $-18,918611$ \\
\hline Southeast & Espírito Santo (ES) & Cach. de Itapemirim & $-41,112778$ & $-20,848889$ \\
\hline Southeast & Espírito Santo (ES) & Cariacica & $-40,420000$ & $-20,263889$ \\
\hline Southeast & Espírito Santo (ES) & Colatina & $-40,630556$ & $-19,539444$ \\
\hline Southeast & Espírito Santo (ES) & Serra & $-40,307778$ & $-20,128611$ \\
\hline Southeast & Espírito Santo (ES) & Viana & $-40,496111$ & $-20,390278$ \\
\hline Southeast & Espírito Santo (ES) & Vila Velha & $-40,292500$ & $-20,329722$ \\
\hline Southeast & Espírito Santo (ES) & Vitória & $-40,337778$ & $-20,319444$ \\
\hline Southeast & Rio de Janeiro (RJ) & Cabo Frio & $-42,018611$ & $-22,879444$ \\
\hline Southeast & Rio de Janeiro (RJ) & C. dos Goytacazes & $-41,324444$ & $-21,754167$ \\
\hline Southeast & Rio de Janeiro (RJ) & Duque de Caxias & $-43,311667$ & $-22,785556$ \\
\hline Southeast & Rio de Janeiro (RJ) & Itaperuna & $-41,887778$ & $-21,205000$ \\
\hline Southeast & Rio de Janeiro (RJ) & Niterói & $-43,103611$ & $-22,883333$ \\
\hline Southeast & Rio de Janeiro (RJ) & Nova Iguaçu & $-43,451111$ & $-22,759167$ \\
\hline Southeast & Rio de Janeiro (RJ) & Rio de Janeiro & $-43,207500$ & $-22,902778$ \\
\hline Southeast & Rio de Janeiro (RJ) & São Gonçalo & $-43,053889$ & $-22,826944$ \\
\hline Southeast & Rio de Janeiro (RJ) & São João de Meriti & $-43,372222$ & $-22,803889$ \\
\hline
\end{tabular}




\begin{tabular}{|c|c|c|c|c|}
\hline Region & State & City & Longitude & Latitude \\
\hline Southeast & Rio de Janeiro (RJ) & S. José do V. Rio Preto & $-42,924444$ & $-22,151389$ \\
\hline Southeast & Rio de Janeiro (RJ) & Três Rios & $-43,209167$ & $-22,116667$ \\
\hline Southeast & Rio de Janeiro (RJ) & Volta Redonda & $-44,104167$ & $-22,523056$ \\
\hline Southeast & São Paulo (SP) & Araçatuba & $-50,432778$ & $-21,208889$ \\
\hline Southeast & São Paulo (SP) & Barretos & $-48,567778$ & $-20,557222$ \\
\hline Southeast & São Paulo (SP) & Bauru & $-49,060556$ & $-22,314722$ \\
\hline Southeast & São Paulo (SP) & Botucatu & $-48,445000$ & $-22,885833$ \\
\hline Southeast & São Paulo (SP) & Campinas & $-47,060833$ & $-22,905556$ \\
\hline Southeast & São Paulo (SP) & Itapevi & $-46,934167$ & $-23,548889$ \\
\hline Southeast & São Paulo (SP) & Itu & $-47,299167$ & $-23,264167$ \\
\hline Southeast & São Paulo (SP) & Jandira & $-46,902500$ & $-23,527500$ \\
\hline Southeast & São Paulo (SP) & Marília & $-49,945833$ & $-22,213889$ \\
\hline Southeast & São Paulo (SP) & Presidente Prudente & $-51,388889$ & $-22,125556$ \\
\hline Southeast & São Paulo (SP) & Ribeirão Preto & $-47,810278$ & $-21,177500$ \\
\hline Southeast & São Paulo (SP) & Santana de Parnaíba & $-46,917778$ & $-23,444167$ \\
\hline Southeast & São Paulo (SP) & Santos & $-46,333611$ & $-23,960833$ \\
\hline Southeast & São Paulo (SP) & São Carlos & $-47,890833$ & $-22,017500$ \\
\hline Southeast & São Paulo (SP) & São José do Rio Preto & $-49,379444$ & $-20,819722$ \\
\hline Southeast & São Paulo (SP) & São Paulo (Pirituba) & $-46,723611$ & $-23,475000$ \\
\hline Southeast & São Paulo (SP) & São Paulo (Ipiranga) & $-46,642222$ & $-23,543889$ \\
\hline Southeast & São Paulo (SP) & São Sebastião & $-45,409722$ & $-23,760000$ \\
\hline Southeast & São Paulo (SP) & Sorocaba & $-47,458056$ & $-23,501667$ \\
\hline South & Paraná (PR) & Foz do Iguaçu & $-54,588056$ & $-25,547778$ \\
\hline South & Paraná (PR) & Londrina & $-51,162778$ & $-23,310278$ \\
\hline South & Paraná (PR) & Jacarezinho & $-49,969444$ & $-23,160556$ \\
\hline South & Paraná (PR) & Maringá & $-51,938611$ & $-23,425278$ \\
\hline South & Paraná (PR) & Palotina & $-53,840000$ & $-24,283889$ \\
\hline South & Rio Grande do Sul (RS) & Crissiumal & $-54,101111$ & $-27,499722$ \\
\hline South & Santa Catarina (SC) & Florianópolis & $-48,549167$ & $-27,596667$ \\
\hline South & Santa Catarina (SC) & Itapiranga & $-53,712222$ & $-27,169444$ \\
\hline
\end{tabular}

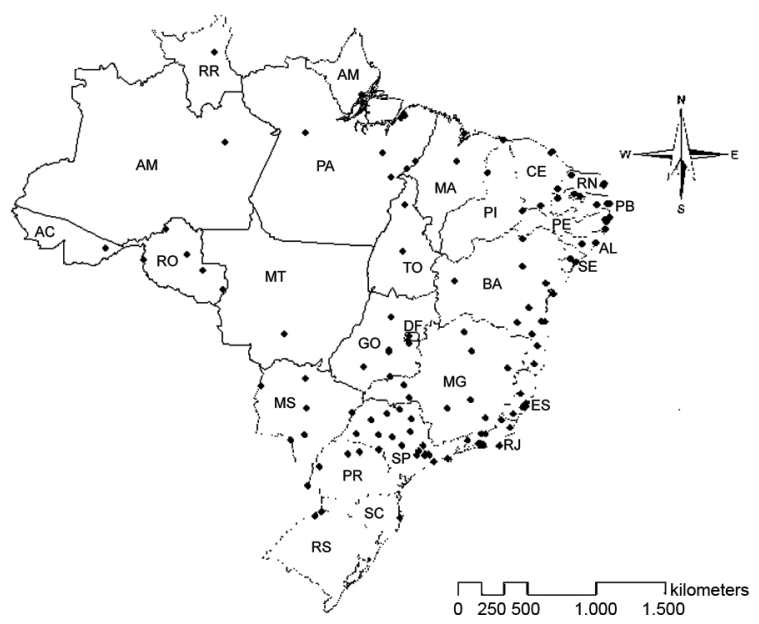

Fig. 1: distribution of the sampling sites of the populations of the yellow fever mosquito Aedes aegypti used in the spatio-temporal survey of temephos resistance in Brazil. Identification for each sampling site and its coordinates are listed in Table I. ers containing temephos-contaminated water (except in the control treatments) and four replicates were used for each locally collected population. Mortality assessment of the mosquito larvae was performed after $24 \mathrm{~h}$ exposure. The larvae were considered dead if they were unable to rise to the surface when dorsally prodded.

Geostatistical analyses - These analyses were based on the geographical coordinates of each mosquito sampling site from which the mosquito populations were obtained and used to calculate the distance between sampling sites. The distances from the sampling sites and the mortality data obtained from the diagnostic bioassays were subjected to alternative kriging methods (stable, circular, spherical, exponential and Gaussian) to select suitable semivariogram functions for distance interpolation (Isaacs \& Srivastava 1989). The semivariogram functions obtained using each group of models allowed the estimation of three parameters to determine their respective shapes: range $\left(\mathrm{h}_{\mathrm{r}}\right)$, partial sill $(\mathrm{C})$, and nugget $\left(\mathrm{C}_{\mathrm{o}}\right)$. The range $\left(\mathrm{h}_{\mathrm{r}}\right)$ and partial sill $(\mathrm{C})$ refer to the point in the semivariogram function in which a plateau 
is reached; the range $\left(\mathrm{h}_{\mathrm{r}}\right)$ corresponds to the distance at which this phenomenon takes place, while the partial sill (C) refers to its respective semivariance value. The nugget $\left(\mathrm{C}_{\mathrm{o}}\right)$ is the semivariogram value in which the model intercepts the $y$-axis (i.e., the mortality semivariance axis) corresponding to measurement errors or spatial sources of variation at distances smaller than the sampling interval (or both). Three additional parameters were calculated from these three basic parameters described above. These were: sill $\left(\mathrm{C}_{\mathrm{o}}+\mathrm{C}\right)$, proportion $[\mathrm{C} /$ $\left.\left(\mathrm{C}_{\mathrm{o}}+\mathrm{C}\right)\right]$ and randomness $\left(\mathrm{C}_{\mathrm{o}} / \mathrm{C}\right)$ of the data. A crossvalidation procedure was subsequently used to select the best data adjustment to compare the observed and estimated data for each sampling point using the model of semivariogram function under test. This estimated error allows the best model selection as those leading to the error average closer to zero, aided by the randomness assessment (the higher, the better). The semivariance data obtained from the selected models were used to generate the spatial maps depicting the phenomenon of temephos resistance. All the spatial analyses were performed using ArcGIS 10 software (ESRI, Redlands, CA, USA).

\section{RESULTS}

General temephos mortality findings - The diagnostic bioassays assessing mosquito larvae mortality by temephos were performed to estimate the frequency of temephos-resistant individuals in the sampled insect populations. This frequency of resistant individuals is indicated as an average mortality score ranging from $80.31 \%$ between $1999-2000$ and dropping to less than 50\% between 2010-2011 (Table II). The number of insect samples tested per year ranged from 25 (from 2010-2011) to 74 (between 2000-2001) and had a broad range of mortality response within each year, resulting in a high standard deviation of larval mortality per year (Table III).

Semivariogram model selection - Suitable semivariogram models were obtained for each biannual dataset of temephos mortality using the diagnostic insecticide resistance bioassays. The selected semivariogram models are exhibited in Table III, along with their respective parameters for model selection. The plots from each model and the respective observed data are exhibited in Fig. 2.

TABLE II

Descriptive statistics of the diagnostic bioassays with temephos on larvae of the yellow fever mosquito Aedes aegypti

\begin{tabular}{|c|c|c|c|c|c|c|c|}
\hline \multirow[b]{2}{*}{ Year } & \multirow{2}{*}{$\begin{array}{l}\text { Sampling sites } \\
\text { (n) }\end{array}$} & \multicolumn{4}{|c|}{ Mortality (\%) } & \multirow{2}{*}{$\begin{array}{c}\text { Skewness } \\
\left(g_{l}\right)\end{array}$} & \multirow{2}{*}{$\begin{array}{l}\text { Kurtosis } \\
\qquad\left(g_{2}\right)\end{array}$} \\
\hline & & Minimum & Maximum & Mean & SD & & \\
\hline 1999-2000 & 64 & 13.15 & 100.00 & 80.31 & 24.62 & -1.22 & 3.40 \\
\hline 2000-2001 & 74 & 10.80 & 100.00 & 71.53 & 26.34 & -0.68 & 2.38 \\
\hline $2002-2003$ & 58 & 2.00 & 99.80 & 62.48 & 30.16 & -0.51 & 2.08 \\
\hline 2004-2005 & 59 & 1.50 & 98.45 & 53.41 & 33.69 & -0.18 & 1.39 \\
\hline 2006-2007 & 39 & 6.40 & 97.60 & 52.33 & 24.48 & -0.16 & 1.97 \\
\hline 2008-2009 & 46 & 6.00 & 96.70 & 50.60 & 24.99 & 0.05 & 1.82 \\
\hline $2010-2011$ & 25 & 7.50 & 88.20 & 49.99 & 28.16 & -0.12 & 1.55 \\
\hline
\end{tabular}

SD: standard deviation

TABLE III

Semivariogram models and parameters of larval mortality by temephos on populations of the yellow fever mosquito Aedes aegypti

\begin{tabular}{|c|c|c|c|c|c|c|c|c|c|}
\hline Year & Kriging & Model & $\begin{array}{c}\text { Nugget } \\
\left(\mathrm{C}_{0}\right)\end{array}$ & $\begin{array}{l}\text { Partial sill } \\
\text { (C) }\end{array}$ & $\begin{array}{c}\text { Sill } \\
\left(\mathrm{C}_{0}+\mathrm{C}\right)\end{array}$ & $\begin{array}{c}\text { Proportion } \\
\left(\mathrm{C} / \mathrm{C}+\mathrm{C}_{0}\right)\end{array}$ & $\begin{array}{l}\text { Range } \\
\left(h_{r}, \mathrm{~m}\right)\end{array}$ & $\begin{array}{l}\text { Randomness } \\
\qquad\left(\mathrm{C}_{0} / \mathrm{C}\right)\end{array}$ & Mean errors \\
\hline $1999-2000$ & Ordinary & Gaussian & 132.963 & 639.079 & 772.042 & 0.827778 & 593820.368 & 0.208054 & -0.027 \\
\hline $2000-2001$ & Simple & Gaussian & 231.740 & 640.182 & 871.922 & 0.734219 & 632424.376 & 0.361991 & -0.059 \\
\hline $2002-2003$ & Simple & Exponential & 391.601 & 972.709 & 1364.31 & 0.712968 & 3658678.194 & 0.402588 & -0.203 \\
\hline 2004-2005 & Ordinary & Gaussian & 224.524 & 176.033 & 400.557 & 0.439471 & 695175.201 & 1.275465 & 0.101 \\
\hline 2006-2007 & Ordinary & Exponential & 162.384 & 669.389 & 831.773 & 0.804774 & 1175553.465 & 0.242585 & -0.096 \\
\hline 2008-2009 & Ordinary & Circular & 57.218 & 723.989 & 781.207 & 0.926757 & 947927.124 & 0.079032 & 0.266 \\
\hline $2010-2011$ & Ordinary & Circular & 367.832 & 262.731 & 630.563 & 0.416661 & 507101.080 & 0.714269 & 1.576 \\
\hline
\end{tabular}



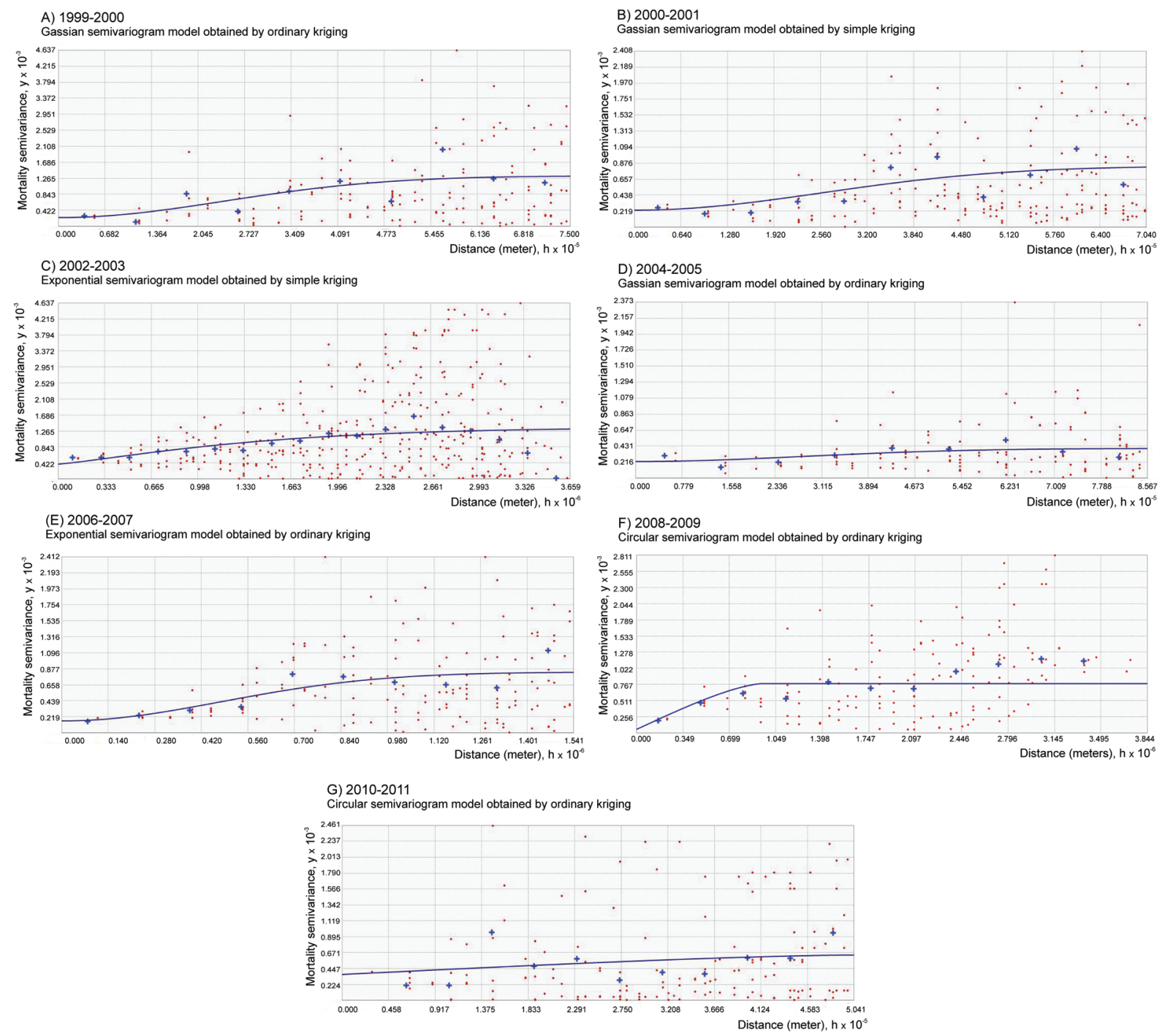

Fig. 2: semivariogram models [mortality semivariance (y) as a function of distance (x)] exhibited in Table II and obtained from the diagnostic bioassays of temephos resistance on larvae of the yellow fever mosquito Aedes aegypti. Observed points are represented as red symbols, and averages are represented as blue crosses.

Temporal spread of temephos resistance - Spatial interpolation using kriging allowed mapping the countrywide spread of temephos resistance in larvae of YF mosquitoes from 1999-2000 until 2010-2011, which is the last year the survey data were available. Initially the efficacy of temephos was high, causing larval mortality of $>80 \%$ throughout Brazil, except in the coastal area, which spans from Pará in the north to Piauí in the northeast and encompasses the state of Rio de Janeiro and neighboring parts of São Paulo and Minas Gerais (Fig. 3). However, the frequency of temephos resistant individuals in the insect populations increased steadily during each biannual survey, reflecting a significant reduction in temephos efficacy. This trend reached high levels $(<50 \%$ mortality $)$ in about half the country as early as 2004-2005 (Fig. 3). Although the frequency of temephos resistance seems to have been attenuated in the main problem areas observed between 20042005 , temephos resistance continued to spread within Brazil. By 2010-2011 only Rondônia (in the North), São Paulo (Southeast), Paraná and Santa Catarina (South) exhibited satisfactory temephos efficacy against YF mosquito larvae. New focal areas of temephos resistance were detected in the 2010-2011 survey radiating from near Rio Branco (southern Acre in North Brazil, near Bolivia) and Brasilia (Central Brazil), leading to a country-wide resistance phenomenon.

\section{DISCUSSION}

The temephos mortality dataset obtained from the diagnostic bioassays performed by the MoReNAa, although not carried out with the objective of spatial in- 

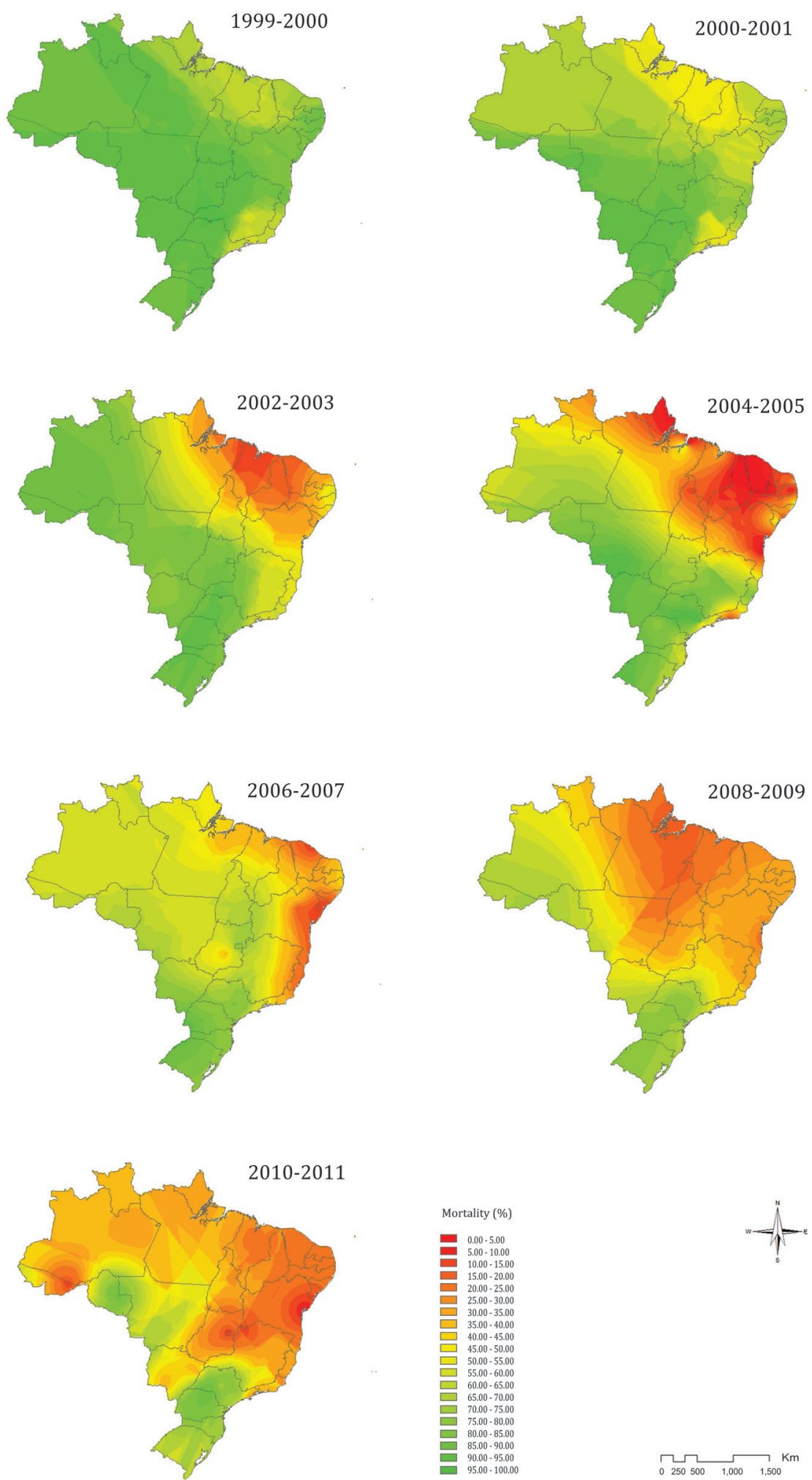

Fig. 3: contour maps of temephos resistance in Brazilian populations of the yellow fever mosquito (Aedes aegypti) generated using spatial interpolation. The colour legend indicates the represented range of mortality (\%) of mosquito larvae obtained in the temephos resistance diagnostic bioassays. Colours tending toward red indicate lower larval mortality and, consequently, a higher frequency of temephos resistance. 
terpolation to generate temephos resistance maps for Brazilian populations of Ae. aegypti, allowed such interpolations and the inferences necessary to generate the maps. The effort provided a means to clearly illustrate the temporal spread and spatial reach of temephos resistance in Ae. aegypti - which greatly increased during the 12-year period of assessment - within the Brazilian territory. Nonetheless, a more fine-tuned survey focusing on diagnostic bioassays of insecticide resistance using larger and better-distributed sampling sites would allow even more comprehensive assessments for eventual decisionmaking regarding policies and procedures to be adopted.

Temephos resistance among Brazilian populations of the YF mosquito is far from novel. All the Brazilian states have adopted the routine use of temephos ( $1 \%$ sand granule formulations) to manage Ae. aegypti by controlling its larvae since the early 1990's (Funasa 1994, 2001, Sucen 1997). The result of this continuous and consistent use of temephos throughout the country led to reports of temephos resistance as early as 1995 (Macoris et al. 1995). The increased incidence of dengue during the 1990s in Brazil attributed to the spread of Ae. aegypti enhanced concern regarding insecticide use against the mosquito and the susceptibility of mosquito populations (da Silva Jr et al. 2002, Braga \& Valle 2007). The end result was the establishment of an insecticide-resistance monitoring program in the country that focused on populations of the YF mosquito (Braga $\&$ Valle 2007). Consistent detection of temephos resistance in different parts of the country soon followed (Campos \& Andrade 2003, Lima et al. 2003, 2006, Macoris et al. 2003, 2007, Melo-Santos et al. 2010, Gambarra et al. 2013, Diniz et al. 2014).

Some of the studies of temephos resistance among Brazilian populations of the YF mosquito explored the mechanisms involved and the existence of fitness costs associated with this resistance. Fitness costs were indeed detected (Diniz et al. 2014). Unfortunately, the studies on the underlying mechanisms of temephos resistance were more confused and patchy, but they were suggestive of the prevailing involvement of enhanced insecticide detoxification as the main mechanism, with esterases likely playing a major role, although not an exclusive one (Braga \& Valle 2007, Melo-Santos et al. 2010, Gambarra et al. 2013, Macoris et al. 2014). These findings seem consistent with mechanistic studies of temephos resistance performed with other Latin American populations of the same species (Bisset Lazcano et al. 2009, Bisset et al. 2013, Grisales et al. 2013, Reyes-Solis et al. 2014, Saavedra-Rodriguez et al. 2014).

The twelve-year effort of the MoReNAa achieved a great deal, but no summary of the country-wide survey effort had ever been performed; therefore, creating such a summary was the objective of the current work. The geostatistical tools used here allowed the recognition of both the temporal pattern of the spread of temephos resistance in the country and its gravity by 2011 . Despite the early detection of temephos resistance in the mid-1990s, country-wide use of temephos continued; consequently, temephos resistance spread throughout the country during the following years, reaching serious levels by
2002-2003. At this point, nearly half of the country was already having problems because of temephos resistance in Ae. aegypti, particularly when considering "resistant" to mean mosquito populations that exhibit mortality levels below the $80 \%$ threshold-a threshold that incurs in a high likelihood of control failure (Davidson \& Zahar 1973). The scenario has simply gotten worse in subsequent years. Now, nearly all the country (except a part in the South) exhibits temephos resistance.

The use of temephos as a mosquito larvicide in Brazil has been suppressed since late in 2010, which may reverse the spread of resistance and allow for future use of the compound. However, the high frequency of resistant individuals already established in the country potentially limits the extent of such (future) use, even if the fitness cost associated with temephos resistance prevails in the country. Effective, safe and cheap insecticides such as temephos, which are the underlying reasons for its global use as a mosquito larvicide, are hard to come by (Tomé et al. 2014). A few alternatives have emerged and are currently being explored, including a few pyrethroids, but these already exhibit insecticide resistance problems in wide areas in Brazil (e.g., Brito et al. 2013). More recently, insect growth regulators and the bioinsecticide Bacillus thuringiensis serovar israelensis (Bti) have been explored (Braga \& Valle 2007, Fontoura et al. 2012, Araújo et al. 2013).

In conclusion, temephos resistance in Brazilian populations of the YF mosquito spread during the 12-year survey period, showing that resistance is now widespread and there is little hope of achieving effective mosquito control with this insecticide. Alternative insecticides aided by the preventive elimination of potential mosquito breeding sites are necessary. However, the use of these alternative insecticides will also lead to the eventual emergence of resistant mosquito populations - and may have already occurred in the country, considering their present rate of use. Therefore, continuing country-wide surveys are necessary to guide management decisions by the national health officers. In such a context, planned yearly systematic sampling and insecticide resistance diagnostic bioassays are necessary. Moreover, geostatistical analyses to map the levels and spread of the phenomenon are also necessary.

\section{ACKNOWLEDGEMENTS}

The authors would like to thank OPAS that allowed the National Insecticide Susceptibility Monitoring Program to be conducted and for making that data available to the present study.

\section{REFERENCES}

Alves SN, Tibúrcio JD, de Melo AL. Susceptibilidade de larvas de Culex quinquefasciatus a diferentes inseticidas. Rev Soc Bras Med Trop. 2011; 44(4): 486-489.

Amorim LB, Helvecio E, de Oliveira CMF, Ayres CFJ. Susceptibility status of Culex quinquefasciatus (Diptera: Culicidae) populations to the chemical insecticide temephos in Pernambuco, Brazil. Pest Manag Sci. 2013; 69(12): 1307-1314.

Araújo AP, Araújo DFD, Helvecio E, Barros RA, Oliveira CMF, Ayres CFJ, et al. The susceptibility of Aedes aegypti populations displaing temephos resistance to Bacillus thuringiensis israelensis: a basis for management. Parasit Vectors. 2013; 6: 297. 
Bisset JA, Marín R, Rodríguez MM, Severson DW, Ricardo Y, French L, et al. Insecticide resistance in two Aedes aegypti (Diptera: Culicidae) strains from Costa Rica. J Med Entomol. 2013; 50(2): 352-361.

Bisset Lazcano JA, Rodríguez MM, San Martín JL, Romero JE, Montoya R. Evaluación de la resistencia a insecticidas de una cepa de Aedes aegypti de El Salvador. Rev Panam Salud Publica. 2009; 26(3): 229-234.

Braga IA, Lima JBP, Soares SS, Valle D. Aedes aegypti resistance to temephos during 2001 in several municipalities in the states of Rio de Janeiro, Sergipe, and Alagoas, Brazil. Mem Inst Oswaldo Cruz. 2004; 99(2): 199-203.

Braga IA, Valle D. Aedes aegypti: vigilância, monitoramento da resistência e alternativas de controle no Brasil. Epidemiol Serv Saude. 2007; 16(4): 295-302.

Brito LP, Linss JGB, Lima-Camara TN, Belinato TA, Peixoto AA, Lima JBP, et al. Assessing the effects of Aedes aegypti kdr mutations on pyrethroid resistance and its fitness cost. PLoS ONE. 2013; 8(4): e60878.

Campos J, Andrade CFS. Susceptibilidade larval de populações de Aedes aegypti e Culex quinquefasciatus a inseticidas químicos. Rev Saude Publica. 2003; 37(4): 523-527.

da Silva Jr J, Siqueira Jr JB, Coelho GE, Vilarinhos PT, Pimenta Jr FG. Dengue in Brazil: current situation and control activities. Epidemiol Bull. 2002; 23(1): 3-6.

Davidson G, Zahar AR. The practical implications of resistance in malaria vectors to insecticides. Bull World Health Organization. 1973; 49(5): 475-483.

Diniz MMCSL, Henriques ADS, Leandro RS, Aguiar DL, Beserra EB. Resistance of Aedes aegypti to temephos and adaptive disadvantages. Rev Saude Publica. 2014; 48(5): 775-782.

Fay RW, Eliason DA. A preferred oviposition site as a surveillance method for Aedes aegypti. Mosq News. 1966; 26(4): 531-534.

Fontoura NG, Bellinato DF, Valle D, Lima JBP. The efficacy of a chitin synthesis inhibitor against field populations of organophosphate-resistant Aedes aegypti in Brazil. Mem Inst Oswaldo Cruz. 2012; 107(3): 387-395.

Funasa - Fundação Nacional de Saúde. Dengue - Instruções para pessoal de combate ao vetor: Manual de Normas Técnicas, 3rd ed. Brasília: Ministério da Saúde; 2001.

Funasa - Fundação Nacional de Saúde. Manual de normas técnicas - Instruções para pessoal de operações. Brasília: Ministério da Saúde; 1994.

Funasa - Fundação Nacional de Saúde. Programa nacional de controle da dengue. Brasília: Ministério da Saúde; 2002.

Funasa - Fundação Nacional de Saúde. Reunião técnica para discutir status de resistência de Aedes aegypti e definir estratégias a serem implantadas para monitoramento da resistência no Brasil. Brasília: Ministério da Saúde; 1999.

Gambarra WPT, Martins WFS, de Lucena Filho ML, de Albuquerque IMC, Apolinário OKS, Beserra EB. Spatial distribution and esterase activity in populations of Aedes (Stegomyia) aegypti (Linnaeus) (Diptera: Culicidae) resistant to temephos. Rev Soc Bras Med Trop. 2013; 46(2): 178-184.

Grisales N, Poupardin R, Gomez S, Fonseca-González I, Ranson H, Lenhart A. Temephos resistance in Aedes aegypti in Colombia compromises dengue vector control. PLoS Negl Trop Dis. 2013; 7(9): e2438.

Gubler DJ. The changing epidemiology of yellow fever and dengue, 1900 to 2003: full circle? Comp Immunol Microbiol Infect Dis. 2004; 27(5): 319-330.

Gubler DJ. The economic burden of dengue. Am J Trop Med Hyg. 2012; 86(5): 743-744.
Guedes RNC, Smagghe G, Stark JD, Desneux N. Pesticide-induced stress in arthropod pests for optimized integrated pest management programs. Annu Rev Entomol. 2016; 61: 43-62.

Guzman MG, Halstead SB, Artsob H, Buchy P, Farrar J, Gubler DJ, et al. Dengue: a continuing global threat. Nat Rev Microbiol. 2010; 8(Suppl. 12): S7-S18.

Halstead SB. Dengue vaccine development: a 75\% solution? Lancet. 2012; 380(9853): 1535-1536.

Hay S. Football fever could be a dose of dengue. Nature. 2013; 503(7477): 439.

Hsieh YH, Chen CWS. Turning points, reproduction number, and impact of climatological events for multi-waver dengue outbreaks. Trop Med Int Health. 2009: 14(6): 628-638.

Isaacs EH, Srivastava RM. An introduction to applied geostatistics. New York: Oxford University Press; 1989.

Jakob WL, Bevier GA. Application of ovitraps in the US Aedes aegypti eradication program. Mosq News. 1969; 29(1): 55-61.

Lima EP, de Oliveira Filho AM, Lima JWO, Ramos Jr NA, Cavalcanti LPG, Pontes RJS. Resistência do Aedes aegypti ao temefós em municípios do estado do Ceará. Rev Soc Bras Med Trop. 2006; 39: 259-263.

Lima EP, Paiva MH, de Araújo AP, da Silva EV, da Silva UM, de Oliveira IN, et al. Insecticide resistance in Aedes aegypti populations from Ceará, Brazil. Parasit Vectors. 2011; 4: 5.

Lima JBP, da Cunha MP, da Silva RC, Galardo AKR, Soares SS, Braga IA, et al. Resistance of Aedes aegypti to organophosphates in several municipalities in the states of Rio de Janeiro and Espírito Santo, Brazil. Am J Trop Med Hyg. 2003; 68(3): 329-333.

Lourenço-de-Oliveira R, Vazeille M, de Filippis AMB, Failloux AB. Aedes aegypti in Brazil: genetically-differentiated populations with high susceptibility to dengue and yellow fever viruses. Trans R Soc Trop Med Hyg. 2004; 98(1): 43-54.

Lowe R, Barcellos C, Coelho CAS, Bailey T, Coelho GE, Graham $\mathrm{R}$, et al. Dengue outlook for the World Cup in Brazil: an early warning model framework driven by real-time seasonal climate forecasts. Lancet Infect Dis. 2014; 14(7): 619-626.

Maciel-de-Freitas R, Avendanho FC, Santos R, Sylvestre G, Araújo $\mathrm{SC}$, Lima JBP, et al. Undesirable consequences of insecticide resistance following Aedes aegypti control activities due to a dengue outbreak. PLoS ONE. 2014; 9(3): e92424.

Macoris MLG, Andrighetti MTM, Nalon KCR, Garbeloto VC, Caldas Jr AL. Standardization of bioassays for monitoring resistance to insecticides in Aedes aegypti. Dengue Bull. 2005; 29: 176-182.

Macoris MLG, Andrighetti MTM, Otrera VCG, de Carvalho LR, Caldas Jr AL, Brogdon WG. Association of insecticide use and alteration on Aedes aegypti susceptibility status. Mem Inst Oswaldo Cruz. 2007; 102(8): 895-900.

Macoris MLG, Andrighetti MTM, Takaku L, Glasser CM, Garbeloto VC, Bracco JE. Resistance of Aedes aegypti from the state of São Paulo, Brazil, to organophosphate insecticides. Mem Inst Oswaldo Cruz. 2003; 98(5): 703-708.

Macoris MLG, Andrighetti MTM, Wanderley DMV, Ribolla PEM. Impact of insecticide resistance on the field control of Aedes aegypti in the state of São Paulo. Rev Soc Bras Med Trop. 2014; 47(5): 573-578.

Macoris MLG, Camargo MF, Silva IG, Takaku L, Andrighetti MTM. Modificação da susceptibilidade de Aedes (Stegomyia) aegypti ao temefós. Rev Pat Trop. 1995; 24(1): 31-40.

Mazzari MB, Georghiou GP. Characterization of resistance to organophosphate, carbamate, and pyrethroid insecticides in field 
populations of Aedes aegypti from Venezuela. J Am Mosq Control Assoc. 1995; 11: 315-322.

Melo-Santos MAV, Varjal-Melo JJM, Araújo AP, Gomes TCS, Paiva MHS, Regis LN, et al. Resistance to the organophosphate temephos: mechanisms, evolution, and reversion in na Aedes aegypti laboratory strain from Brazil. Acta Trop. 2010; 113: 180-189.

OPAS - Organización Panamericana de la Salud. Dengue y dengue hemorrágico en las Américas: guías para su prevención y control. Washington DC: OPS; 1995.

Phophiro JS, Silva OS, Luna JED, Piccoli CF, Kanis LA, da Silva MAN. Aedes aegypti and Aedes albopictus (Diptera: Culicidae): coexistence and susceptibility to temephos, in municipalities with occurrence of dengue and differentiated characteristics of urbanization. Rev Soc Bras Med Trop. 2011; 44(3): 300-305.

Rawlins SC, Wan JO. Resistance in some Caribbean populations of Aedes aegypti to several insecticides. J Am Mosq Control Assoc. 1995; 11(1): 59-65.

Reyes-Solis GC, Saavedra-Rodriguez K, Suarez AF, Black 4th WC. QTL mapping of genome regions controlling temephos resistance in larvae of the mosquito Aedes aegypti. PLoS Negl Trop Dis. 2014; 8(10): e3177.

Saavedra-Rodriguez K, Strode C, Flores AE, Garcia-Luna S, ReyesSolis G, Ranson H, et al. Differential transcription profiles in Ae- des aegypti detoxification genes after temephos selection. Insect Mol Biol. 2014; 23: 199-215.

Schatzmayr HG. Dengue situation in Brazil by year 2000. Mem Inst Oswaldo Cruz. 2000; 95(Suppl. 1): S179-S181.

Sucen - Superintendência de Controle de Endemias. Plano de erradicação de Aedes aegypti: Guia de instruções. São Paulo: Secretaria de Estado da Saúde de São Paulo; 1997.

Tomé HVV, Pascini TV, Dângelo RAC, Guedes RNC, Martins GF. Survival and swimming behavior of insecticide-exposed larvae and pupae of the yellow fever mosquito Aedes aegypti. Parasit Vectors. 2014; 7: 195.

van Panhuis WG, Hyun S, Blaney K, Marques Jr ETA, Coelho GE, Siqueira Jr JB, et al. Risk of dengue for tourists and teams during the World Cup 2014 in Brazil. PLoS Negl Trop Dis. 2014; 8(7): e3065.

WHO - World Health Organization. Expert committee on vector biology and control. Vector resistance to pesticides: WHO Technical Report Series 818. Geneva: WHO; 1992.

WHO - World Health Organization. Global insecticide use for vector-borne disease control. 4th ed. Geneva: WHO/HTM/NTD/ WHOPES/GCDPP; 2009 .

WHO - World Health Organization. Instruction for determining the susceptibility or resistance of mosquito larvae to insecticides. Geneva: WHO/VBC; 1981. 